\title{
Update on duloxetine for the management of stress urinary incontinence
}

\author{
Maya Basu \\ Jonathan RA Duckett
}

Department of Obstetrics and Gynaecology, Medway Maritime Hospital, Gillingham, Kent, UK
Correspondence: Jonathan RA Duckett Consultant Gynaecologist, Medway Maritime Hospital, Windmill Road, Gillingham, Kent, UK, ME7 5NY

$\mathrm{Tel}+44$ I634 825I54

Fax +44 I634 825I55

Email jraduckett@hotmail.com

\begin{abstract}
Duloxetine is a relatively balanced serotonin and noradrenaline reuptake inhibitor (SNRI), which is the first drug with widely proven efficacy to have been licensed for the medical treatment of women with stress urinary incontinence (SUI). Despite favorable results from randomized controlled trials, surgical management continues to be the mainstay of treatment for SUI. In this review we explore the pharmacology of duloxetine in the nervous system and lower urinary tract, and the evidence for its use in the management of women with urinary incontinence.
\end{abstract}

Keywords: duloxetine, stress urinary incontinence, overactive bladder

\section{Introduction}

Urinary incontinence is a highly prevalent chronic medical condition which has a known negative impact on quality of life in women. It can impact significantly on physical activities, confidence and self-perception and social activities. ${ }^{1}$ There is also a known association between lower urinary tract symptoms (LUTS) and female sexual dysfunction. ${ }^{2}$ Urinary incontinence may be further categorized as stress urinary incontinence (SUI), urge urinary incontinence (UUI) or mixed urinary incontinence (MUI). SUI is defined as the complaint of involuntary leakage of urine upon effort or exertion, or on sneezing or coughing. ${ }^{3}$ SUI is estimated to affect $78 \%$ of incontinent women with $29 \%$ complaining of SUI in combination with urge incontinence, and $49 \%$ complaining of pure SUI. Despite the prevalence of stress urinary incontinence and the significant impact that it can have on all domains of quality of life and personal relationships, it is estimated that only a third of women ever seek medical advice concerning their incontinence. ${ }^{4}$ Reasons for this reticence are varied and include embarrassment, lack of knowledge of available treatment options and the belief that incontinence is an inevitable consequence of childbirth or ageing. Unfortunately lack of awareness also exists amongst primary care physicians, with management often being based purely on containment products. This has considerable economic considerations, with one study estimating pad costs in women with incontinence at US\$750 per woman per year. ${ }^{5}$

Management of women with SUI is initially conservative with a period of pelvic floor muscle training. Until recently, the next treatment option offered to women with persistent symptoms was a surgical procedure to correct the anatomical defect. This is now most commonly a suburethral sling, either retropubically or via a transobdurator approach. Long term data on the most established suburethral sling (Tension Free Vaginal Tape, TVT) has shown it to be a highly effective treatment for stress leakage with success rates of $81 \%$ at 5 years. ${ }^{6}$ This treatment escalation from conservative management directly to surgical management reveals a distinct gap for effective medical treatments. Medications such as estrogens and tricyclic antidepressants have been used off label in the past for SUI with little or no evidence of efficacy and often significant side effects. The introduction of the serotonin and noradrenaline reuptake inhibitor (SNRI) duloxetine as a drug therapy for 
stress incontinence was hailed as a significant step forward in terms of widening treatment options for women with SUI. It was the first available pharmacological option for SUI which had proven efficacy in randomized controlled trials, thus filling the treatment void between conservative and surgical therapies. In this article, we discuss the mechanism of action of duloxetine in the lower urinary tract and central nervous system, the original uses of duloxetine in psychiatric disorders and chronic pain, and the available evidence regarding the use of duloxetine in women with incontinence.

\section{Duloxetine in the management of depression and pain}

Duloxetine was originally developed for use in patients with major depressive disorder and more recently, chronic pain. It has been shown in a number of studies that there is a considerable overlap of both incontinence and chronic pain symptoms in subjects with depression. ${ }^{7}$ It was only once the wide-ranging roles of serotonin and noradrenaline in the central and peripheral nervous systems were understood that a common neurobiochemical etiology could be appreciated, and the dual efficacy of duloxetine in depression and pain plus incontinence could be explained. High concentrations of serotonin and noradrenaline terminals are found in the spinal cord, particularly the superficial dorsal/posterior horns. Serotonin and noradrenaline have been shown to be critical to the modulation of pain perception via their effects on inhibitory pain pathways. ${ }^{8}$ The pathophysiology of persistent pain is poorly understood, however there is increasing evidence that imbalance of noradrenaline and serotonin based pathways may play a major role. ${ }^{9}$ Noradrenaline and serotonin terminals are also widespread in higher brain centers responsible for regulation of emotions and mood, particularly the limbic system. A deficiency in one or more monoamine neurotransmitters can lead to the development of anxiety and clinical depression. ${ }^{10}$ This is the basis for the use of SNRIs such as duloxetine in patients with clinical depression. Duloxetine is thought to increase the availability of serotonin and noradrenaline in the synaptic cleft in centers involved in the regulation of mood and emotions. It has been shown to be a safe and effective treatment in several randomized controlled trials. ${ }^{11-13}$ Similarly, duloxetine has been the subject of study as a treatment for chronic pain due to diabetic peripheral neuropathy and has had its efficacy and safety proven in further randomized controlled trials. ${ }^{14}$

\section{Effect of duloxetine on the lower urinary tract}

SUI is thought to arise either due to poor functioning of the striated muscle of the urethral sphincter (instinsic sphincter deficiency, ISD) $)^{15}$ or urethral hypermobility due to loss of vaginal support structures. ${ }^{16}$ It is increasingly believed that both may co-exist to some degree in the majority of women with SUI. Although interventions such as suburethral slings correct the hypermobility of the urethra, duloxetine is thought to act on the sphincter muscle itself.

The neurological control of the lower urinary tract has been elegantly studied in animal models, giving us insight into the role of neurotransmitters, both centrally and peripherally. The micturition cycle consists of a storage phase and a voiding phase. During the storage phase, the detrusor muscle relaxes to accommodate urine, whilst the outlet resistance is enhanced via increased tone of the rhabdosphincter, pelvic floor and urethral smooth muscle. This mechanism is thought to be mediated by cholinergic mechanisms. It has been demonstrated in animal and human studies that the pelvic floor has a nerve supply from branches of the levator ani nerve (from sacral nerve roots S3-S5). ${ }^{17,18}$ One may assume that activity of these nerves causes pelvic floor contraction. The overall process is co-ordinated by the central nervous system with glutamate being the main neurotransmitter for the storage phase. Glutamate is released to Onuf's nucleus (in the ventral horn of the sacral spinal cord), which activates the pudendal nerve, causing a release of acetylcholine and eliciting contraction of the rhabdosphincter. ${ }^{19}$ Both noradrenaline and serotonin are thought to have a modulatory effect on rhabdosphincter contraction, with enhanced activity of the sphincter fibres in their presence. Onuf's nucleus is known to be densely populated with serotonin and noradrenaline receptors and terminals which further implies an important role for these neurotransmitters in the control of the lower urinary tract. It should be noted however that noradrenaline and serotonin are unable to induce rhabdosphincter contraction in the absence of glutamate, ${ }^{20}$ implying that glutamate is the ultimate controller of motor activity, with serotonin and noradrenaline merely potentiating its effects. During the voiding phase of the micturition cycle, glutamate release to Onuf's nucleus ceases, leading to cessation of pudendal nerve activity. Again, this is independent of serotonin and noradrenaline.

The effects of duloxetine are thought to be mediated by an increase in the availability of serotonin and noradrenaline in the synaptic clefts of Onuf's nucleus.

This was first studied by Thor and colleagues in a cat model of bladder irritation. ${ }^{21}$ Administration of duloxetine lead to a dose dependent increase in bladder capacity and enhanced periurethral striated muscle activity. Duloxetine did 
not affect bladder contractions caused by direct electrical stimulation of efferent fibres in the pelvic nerve, leading to the supposition that its effects are due to central rather than peripheral mechanisms. These early studies in animals lead to mechanistic studies in human subjects. Boy et al demonstrated a lower motor threshold for sphincter contraction in response to cortical stimulation and increased pressure amplitudes in response to sacral magnetic stimulation, supporting the conclusion that duloxetine facilitates a positive neuromodulatory effect on the urethral sphincter. ${ }^{22}$ Another study of duloxetine versus placebo in women with SUI showed a trend towards an increase in Valsalva leak point pressure and urethral pressure profile parameters in women taking duloxetine. ${ }^{23}$ A study of the effect of duloxetine on urethral pressure and sphincter morphology showed significant increases in mean urethral retro-resistance pressure, maximum urethral closure pressure and sphincter width after 8 weeks' treatment with duloxetine. ${ }^{24} \mathrm{~A}$ sub-analysis of women who had a symptomatic improvement with duloxetine revealed these increases to be even more marked. Another ultrasound study has confirmed that duloxetine leads to a significant increase in the width of the smooth muscle component of the urethral sphincter, with no corresponding increase in urethral length, ${ }^{25}$ which raises the interesting prospect that duloxetine leads to enhanced activity of smooth as well as striated muscle. The available evidence would seem to support the conclusion that duloxetine improves the symptom of SUI by increasing urethral closure pressure and potentiating the electrical activity of the sphincter.

\section{Management of SUI with duloxetine}

These early studies have been supported by several large phase 2 and 3 trials, which aimed to more clearly delineate the efficacy and safety of duloxetine in women with SUI. One of the first was a double blind randomized placebo controlled study in which 553 women with SUI received placebo, $20 \mathrm{mg}$ duloxetine, $40 \mathrm{mg}$ duloxetine or $80 \mathrm{mg}$ duloxetine for 12 weeks. ${ }^{26}$ The authors reported significant, dose dependent improvements in quality of life and incontinence episode frequency (IEF) and an increase in average voiding interval in women taking duloxetine. There was a high placebo response, with a $41 \%$ decrease in IEF in the placebo group (compared to a $54 \%$ decrease in the duloxetine $20 \mathrm{mg}$ group). Similar results were seen in a subgroup of women with severe SUI. Discontinuation rates for adverse events were 9, 12 and $15 \%$ in the 20, 40 and $80 \mathrm{mg}$ groups respectively, versus $5 \%$ in the placebo group. The most common adverse event was nausea.
Duloxetine was further evaluated in several phase 3 trials. A 2003 trial which randomized 683 North American women to placebo, $80 \mathrm{mg}$ duloxetine once daily or $40 \mathrm{mg}$ duloxetine twice daily over a 12 week period showed duloxetine to cause a significant decrease in median IEF ( $57 \%$ vs $27 \%$, p < 0.001$)$ and a significant improvement in quality of life scores $(11.0$ vs $6.8, \mathrm{p}<0.001) .{ }^{27}$ This study also addressed the question of whether the benefits experienced by women on duloxetine may be due to treatment of underlying depressive symptoms, by using a validated depression inventory as an outcome measure; this demonstrated no significant change in depressive symptoms after treatment with either duloxetine or placebo. $24 \%$ of women in the duloxetine group discontinued the medication, most commonly due to nausea. These findings were subsequently confirmed in another phase 3 trial involving 494 European and Canadian women, which again showed a significant decrease in median IEF for women taking duloxetine versus placebo (50\% vs $29 \%, \mathrm{p}=0.002) .{ }^{28}$ Again, nausea was experienced by $27.9 \%$ of the treatment group (vs $6.5 \%$ in the placebo group, $\mathrm{p}<0.001$ ), although $83 \%$ of the patients who developed nausea whilst on duloxetine did complete the study. A third phase 3 trial of women across 4 continents found similar results, ${ }^{29}$ although with a higher placebo response (40\%) than in the previous phase 3 studies. The authors attribute this to a possibly higher number of treatment naïve subjects in this trial, owing to the diverse geographical regions in which patients were recruited.

Overall, these 3 parallel phase 3 studies randomized 1635 women with SUI worldwide to receive duloxetine in various doses or placebo. Each study consistently showed duloxetine to lead to appreciable decreases in IEF (50\%-54\%) with improvements in quality of life and significant increases in voiding intervals, indicating that the reduction in incontinence episodes was not merely due to learned behaviour developed over the course of the trials. These results were reinforced by a trial which showed duloxetine to be more effective than pelvic floor muscle training or no treatment, and that duloxetine and pelvic floor muscle training had an additive effect when used together. ${ }^{30}$ This presumably reflects the complementary modes of action of these therapies.

Based on the assumption that the mode of action of duloxetine involves enhanced activity of the rhadbosphincter, there have been concerns that it may be less efficacious in older women due to age-related changes in the striated portion of the urethral sphincter. ${ }^{31}$ Older patients may also be more prone to developing adverse effects as a result of drug therapy. A pooled data analysis of 4 large randomized 
controlled trials showed women over 65 to have a slightly diminished reduction in IEF, although response differences when compared to placebo were still maintained. ${ }^{32}$ Indeed, in an analysis of factors that may have been likely to reduce patient response to duloxetine (such as age, body mass index, hypo-estrogenism), the only identifiable factor in this paper which predicted a lack of treatment response was chronic lung disease. The issue of age has been studied more directly by Schagen van Leeuwen and colleagues. ${ }^{33}$ In a 12 -week randomized double blind trial of duloxetine versus placebo in women with SUI or stress predominant mixed UI over the age of 65, significant reductions in IEF with duloxetine were seen; the response rate was comparable to that in the phase 3 studies. There was no significant difference in discontinuation rates between the treatment groups, and interestingly the incidence of nausea was not significantly higher in the duloxetine group. The most common adverse effects in this cohort were dry mouth, fatigue, constipation and hyperhidrosis.

These encouraging trial results seem to suggest that duloxetine is a viable and efficacious alternative to surgery. However, there are common limiting factors with each of the trials discussed, which makes it difficult to assess whether the results can be applied to a "real world" situation. Placebo responses of up to $40 \%$ were seen in the initial trials of duloxetine. ${ }^{26-29}$ Although the decreases in IEF seen with duloxetine were found to be significantly different from those occurring with placebo, this does raise the issue of whether the reported symptom improvement in these trials was partially attributable to the non-pharmacological benefits that being in a trial confers, for example regular contact with physicians and continence advisors. In addition, the study period for each of the phase 3 trials was limited to 12 weeks. It could be argued that this does not reflect use of duloxetine outside of a trial situation since women may be more willing to tolerate adverse effects, as they know the time over which they will have to take the medication is limited.

This has been addressed in two papers reporting outcomes in a cohort of women prescribed duloxetine in routine clinical practice, outside of a trial environment. The cohort consisted of 228 women with urodynamic stress incontinence who were prescribed duloxetine as an alternative to surgery. $67 \%$ of the cohort were started on $40 \mathrm{mg}$ duloxetine twice daily, and remained on this dose for the initial 4-week study period. $33 \%$ were treated with an escalating dose $(20 \mathrm{mg}$ twice daily, increasing the $40 \mathrm{mg}$ twice daily after 2 weeks. $68 \%$ of patients discontinued duloxetine within the first 4 weeks of treatment, mainly because of adverse effects.
Discontinuation due to adverse effects was more common in women who were started immediately on the $40 \mathrm{mg}$ twice daily dose compared with the escalating dose. In women who were able to tolerate duloxetine, $67 \%$ reported an improvement in their symptoms, although the overall improvement rate was just $37 \% .{ }^{34}$ It is immediately obvious that the discontinuation rates and overall efficacy reported in this paper are inferior to those found in the pooled phase 3 studies, leading the authors to conclude that the tolerability of duloxetine in a non-trial situation is poorer than one might expect. This initial paper focussed on outcomes after four weeks' treatment, since $100 \%$ response has been seen by day 15 of treatment in previous studies. ${ }^{35}$ Outcomes for the same cohort one year after commencing duloxetine therapy have more recently been reported. ${ }^{36}$ At this point, just $9 \%$ of the cohort continued to take duloxetine, with $82 \%$ having undergone a tension free vaginal tape. Of the women who discontinued duloxetine, $33 \%$ did so because of lack of efficacy, $56 \%$ because of side effects and $11 \%$ because of an unwillingness to take long term medication.

\section{Management of overactive bladder with duloxetine}

The early animal studies of the actions of duloxetine on the lower urinary tract indicated that as well as leading to enhanced periurethral striated muscle activity, it also caused a dose dependent increase in bladder capacity. ${ }^{21}$ This raises the question of whether it has any treatment effects in women with overactive bladder syndrome $(\mathrm{OAB})$ and/or detrusor overactivity (DO). A recently published study comparing duloxetine to placebo in women with OAB symptoms with or without DO, but excluding those with urodynamic evidence of stress incontinence showed duloxetine treatment to cause a significant reduction in voiding episodes and urge incontinence episodes, when compared to placebo. ${ }^{37}$ There were also observable increases in voiding intervals. A further study examined the effect of duloxetine in women with mixed incontinence (MUI), with the classification of the incontinence (for example, urge predominant mixed incontinence) carried out based on either patient reported symptoms using a validated questionnaire or urodynamic studies. The authors found that duloxetine reduced both stress incontinence episodes and urge incontinence episodes ${ }^{38}$ in women with stress predominant MUI and urge predominant MUI respectively. It is interesting to note in this trial that these same benefits were not seen in women with balanced MUI; the authors attribute this to the small numbers of women within this group. These studies and others would 
seem to indicate that the role of serotonin and noradrenaline in control of the lower urinary tract is more widespread than first thought. Certainly they support the hypothesis that SNRIs may provide an alternative therapeutic approach to OAB symptoms; however direct comparative studies of SNRIs and the current standard antimuscarinic treatments would need to be carried out before any firm conclusions can be drawn.

\section{Investigations of other SNRIs in the management of SUI}

Following on from the sizeable amount of evidence concerning the use of duloxetine in women with SUI, there has been interest in exploring other SNRIs, which may have a more favorable side effect profile. The only drug to be studied in any detail so far is venlafaxine, which is another anti-depressant drug. It is used elsewhere in gynecological practice for the management of hot flushes. Animal studies have shown venlafaxine to lead to a significant increase in urethral pressure profile parameters, ${ }^{39}$ which makes it a plausible alternative to duloxetine. This has been explored in a recent small randomized control trial which compared outcomes in 20 women taking venlafaxine to 20 women taking placebo. ${ }^{40}$ The authors report significant decreases in IEF and increases in quality of life in women with SUI taking venlafaxine. $40 \%$ of women in the active treatment group complained of nausea, however none discontinued the trial because of this. Interestingly, there was no significant placebo effect seen in this trial in terms of IEF, quality of life measures, or patient global impression of improvement. The reasons for this are unclear since the trial design and frequency of follow up for this trial were similar to that of the duloxetine phase 2 and 3 trials. Possibly the smaller number of participants in this trial is implicated in this. Futher studies of venlafaxine (including head to head studies with duloxetine) are necessary to determine whether it may be a preferable drug to duloxetine for women with SUI.

\section{Overall role of duloxetine}

The overall place for medical agents such as duloxetine in the management of women with SUI remains unclear. Certainly, clinical guidelines seem to favor surgery over duloxetine, possibly because it is more cost effective. ${ }^{41}$ There is a wealth of good quality evidence from randomized controlled trials supporting significant treatment benefits in women taking duloxetine when compared to placebo. ${ }^{26-29}$ However, this is complicated by a relatively high incidence of adverse effects, most commonly nausea. The studies of duloxetine in a non-trial situation indicate that women are seemingly less willing to put up with adverse effects, leading to a high discontinuation rate. Other randomized studies have overcome this by dose escalation regimes, but again, this has been in the context of a clinical trial for a finite treatment period. ${ }^{42}$ The majority of women may eventually opt for surgical treatment in the form of a TVT or other sub-urethral sling. The trend towards less invasive surgical options in urogynecology certainly makes surgical treatment a less radical and risky option. Whilst duloxetine leads to a $50 \%$ to $54 \%$ reduction in IEF, the TVT is associated with an $81 \%$ subjective cure rate at 5 years. ${ }^{6}$ Results from a study examining patient expectations from treatments for incontinence indicated that $69 \%$ of women find the option of taking regular medications for life unacceptable. ${ }^{43}$ Additionally, the majority of women expect a complete cure of their incontinence, which is more likely to be achieved with a surgical procedure than duloxetine treatment. It does therefore seem logical that most women would choose to have a one-off minimally invasive surgical procedure with a higher success rate, as opposed to taking a medication which is associated with a significant incidence of adverse effects.

The place of duloxetine may therefore be in women who are unfit for surgery or who do not wish to undergo surgery. It may also be used in women with severe incontinence who are awaiting surgery, or in women who have yet to complete their families. Despite the availability of medical and surgical options, it is known that many women never receive treatment for SUI due to various treatment barriers (either economic or psychosocial). Duloxetine is also not licensed for use in all countries, meaning that the option of medical therapy is denied to many women. The challenge for future research is to investigate other agents which may confer similar treatment benefits to duloxetine, with a lower incidence of side effects.

\section{Disclosures}

Neither author has conflicts of interest to disclose.

\section{References}

1. Fultz N, Burgio K, Diokno A, Kinchen K, Odenchain R, Bump R. Burden of stress urinary incontinence for community dwelling women. Am J Obstet Gynecol. 2003;189:1275-1282.

2. Rogers G, Villarreal A, Kammerer-Doak D, Qualls C. Sexual function in women with and without urinary incontinence and/or pelvic organ prolapse. Int Urogynecol J Pelvic Floor Dysfunct. 2001;12:361-365.

3. Abrams P, Cardozo L, Fall M, et al. The standardisation of terminology in lower urinary tract function: report from the standardisation sub-committee of the International Continence Society. Urology. 2003;61:37-49.

4. Hunskaar S, Lose G, Sykes D, Voss S. The prevalence of urinary incontinence in women in four European countries. BJU Int. 2004;93:324-330. 
5. Subak L, Brown J, Kraus S, et al. The "costs" of urinary incontinence for women. Obstet Gynecol. 2006;107:908-916.

6. Ward K, Hilton P. UK and Ireland TVT Trial Group. Tension free vaginal tape versus colposuspension for primary urodynamic stress incontinence: 5 year follow up. BJOG. 2008;115:226-233.

7. Melville J, Delaney K, Newton K, Katon W. Incontinence severity and major depression in incontinent women. Obstet Gynecol. 2005;106:585-592.

8. Fields H, Heinricher M, Mason P. Neurotransmitters in nociceptive modulatory circuits. Annu Rev Neurosci. 1991;14:219-245.

9. Ren K, Dubner R. Descending modulation in persistent pain: an update. Pain. 2002;100:1-6.

10. Stahl S. Basic psychopharmacology of antidepressants, part I: antidepressants have seven distinct mechanisms of action. $J$ Clin Psychiatry. 1998;59(suppl 4):5-14.

11. Detke M, Lu Y, Goldstein D, et al. Duloxetine $60 \mathrm{mg}$ once daily for major depressive disorder: a randomised double-blind placebo controlled trial. J Clin Psychiatry. 2002;63:308-315.

12. Goldstein D, Mallinckrodt C, Lu Y, Demitrack M. Duloxetine in the treatment of major depressive disorder: a double blind clinical trial. J Clin Psychiatry. 2002;63:225-231.

13. Raskin J, Goldstein D, Mallinckrodt C, Ferguson M. Duloxetine in the long term treatment of major depressive disorder. J Clin Psychiatry. 2003;64:1237-1244.

14. Raskin J, Pritchett Y, Wang F, et al. A double blind, randomized multicenter trial comparing duloxetine with placebo in the management of diabetic peripheral neuropathic pain. Pain Med. 2005;6:346-356.

15. Pajoncini C, Constantini F, Guercini F, Bini V, Porena M. Clinical and urodynamic features of intrinsic sphincter deficiency. Neurourol Urodyn. 2003;22:264-268.

16. Fleischmann N, Flisser A, Blaivas J, Panagopoulos G. Sphincteric urinary incontinence: relationship of vesical leak point pressure, urethral mobility and severity of incontinence. J Urol. 2003;169:999-1002.

17. Pierce L, Reyes M, Thor K, et al. Innervation of the levator ani muscles in the female squirrel monkey. Am J Obstet Gynecol. 2003;188:1141-1147.

18. Barber M, Bremer R, Thor K, Dolber P, Kuehl T, Coates K. Innervation of the female levator ani muscles. Am J Obstet Gynecol. 2002;187:64-71.

19. Michel M, Peters $\mathrm{S}$. Role of serotonin and noradrenaline in stress urinary incontinence. BJU Int. 2004;94(Suppl 1):23-30.

20. Espey M, Downie J. Serotonergic modulation of cat bladder function before and after spinal transection. Eur J Pharmacol. 1995;221:167-117.

21. Thor K, Katofiasc M. Effects of duloxetine, a combined serotonin and norepinephrine reuptake inhibitor, on central neural control of lower urinary tract function in the chloralose-anaesthetized female cat. J Pharmacol Exp Ther. 1995;274:1014-1024.

22. Boy S, Reitz A, Wirth B, Karsenty G, Knapp P, Schurch B. Facilitatory neuromodulative effect of duloxetine on urethral sphincter motor neurones in healthy females. Eur Urol Suppl. 2005;4:253. Abstract 998.

23. Bump R, Benson J, Brubaker L, et al. Biomechanical and electrophysiological effects of duloxetine in women with stress urinary incontinence. Neurourol Urodyn. 2004;23:abstract 269.

24. Athanasiou S, Chaliha C, Digesu A, et al. The effects of duloxetine on urethral function and sphincter morphology. Int Urogynecol J Pelvic Floor Dysfunct. 2007;18:763-767.

25. Duckett J, Patil A, Aggarwal I. The effect of duloxetine on urethral sphincter morphology. Ultrasound Obstet Gynecol. 2008;31:206-209.

26. Norton P, Zinner N, Yalcin I, Bump R, for the Duloxetine Urinary Incontinence Study Group. Duloxetine versus placebo in the treatment of stress urinary incontinence. Am J Obstet Gynecol. 2002;187:40-48.
27. Dmochowski R, Miklos J, Norton P, Zinner N, Yalcin I, Bump R, for the Duloxetine Urinary Incontinence Study Group. Duloxetine versus placebo for the treatment of North American women with stress urinary incontinence. J Urol. 2003;170:1259-1263.

28. van Kerrebroeck P, Abrams P, Lange R, et al. for the Duloxetine Urinary Incontinence Study Group. Duloxetine versus placebo in the treatment of European and Canadian women with stress urinary incontinence. BJOG. 2004;111:249-257.

29. Millard R, Moore K, Rencken R, Yalcin I, Bump R, for the Duloxetine Urinary Incontinence Study Group. Duloxetine versus placebo in the treatment of stress urinary incontinence: a four continent randomized clinical trial. BJU Int. 2004;93:311-318.

30. Ghoniem G, Schagen van Leeuwen J, Elser D, et al. A randomized controlled trial of duloxetine alone, pelvic floor muscle training alone, combined treatment and no active treatment in women with stress urinary incontinence. J Urol. 2005;173:1647-1653.

31. Perucchini D, DeLancey J, Ashton-Miller J, Galecki A, Scheer G. Age effects on urethral striated muscle II: anatomic location of muscle loss. Am J Obstet Gynecol. 2002;186:356-360.

32. Viktrup L, Yalcin I. Duloxetine treatment of stress urinary incontinence in women: effects of demographics, obesity, chronic lung disease, hypoestrogenism, diabetes mellitus, and depression on efficacy. Eur J Obstet Gynecol Reprod Biol. 2007;133:105-113.

33. Schagen van Leeuwen J, Lange R, Jonasson A, Chen W, Viktrup L. Efficacy and safety of duloxetine in elderly women with stress urinary incontinence or stress-predominant mixed urinary incontinence. Maturitas. 2008;60:138-147.

34. Duckett J, Vella M, Kavakuntla G, Basu M. Tolerability and efficacy of duloxetine in a non-trial situation. BJOG. 2007;114:543-547.

35. Cardozo L, Drutz H, Baygani S, Bump R. Pharmacological treatment of women awaiting surgery for stress incontinence. Obstet Gynecol. 2004;104:511-519.

36. Vella M, Duckett J, Basu M. Duloxetine one year on: the long term outcome of a cohort of women prescribed duloxetine. Int Urogynecol J Pelvic Floor Dysfunct. 2008;19:961-964.

37. Steers W, Herschorn S, Kreder K, et al. Duloxetine OAB Study Group. Duloxetine compared with placebo for treating women with symptoms of overactive bladder. BJU Int. 2007;100:337-345.

38. Bent A, Gousse A, Hendrix S, et al. Duloxetine compared with placebo for the treatment of women with mixed urinary incontinence. Neurourol Urodyn. 2008;27:212-221.

39. Bae J, Moon D, Lee J. The effects of a selective noradrenaline reuptake inhibitor on the urethra: an in vitro and in vivo study. $B J U$ Int. 2001;88:771-775.

40. Erdinc A, Gurates B, Celik H, Polat A, Kumru S, Sinsek M. The efficacy of venlafaxine in treatment of women with stress urinary incontinence. Arch Gynecol Obstet. 2008; Jul 16. Epub ahead of print.

41. National Collaborating Centre for Women's and Children's Health. NICE Clinical Guideline Number 40: The management of urinary incontinence in women. National Institute for Health and Clinical Excellence 2006, London.

42. Castro-Diaz D, Palma P, Bouchard C, et al. Duloxetine Dose Escalation Study Group. Effect of dose escalation on the tolerability and efficacy of duloxetine in the treatment of women with stress urinary incontinence. Int Urogynecol J Pelvic Floor Dysfunct. 2007;18:919-929.

43. Robinson D, Anders K, Cardozo L, et al. What do women want: interpretation of the concept of cure. J Pelvic Med Surg. 2003;9:273-277. 